\title{
Correction to: Water Resources Management in Balkan Countries
}

\author{
Abdelazim M. Negm, Gheorghe Romanescu and Martina Zelenakova
}

\section{Correction to:}

\section{A. M. Negm et al. (eds.), Water Resources Management in Balkan Countries, Springer Water, https://doi.org/10.1007/978-3-030-22468-4}

This book was inadvertently published with the incorrect country name as "Macedonia". The belated correction updated "Macedonia" to "North Macedonia". This has now been amended throughout the book. 